\title{
ANÁLISIS DEL LIDERAZGO FEMENINO EN DOS INSTITUTOS DE SECUNDARIA DE CASTILLA Y LEÓN DESDE LA PERSPECTIVA DEL ALUMNADO
}

\author{
Sofía Díaz de Greñu Domingo \\ Rocío Anguita Martínez \\ Universidad de Valladolid
}

\begin{abstract}
RESUMEN: La participación de las mujeres en los puestos directivos a nivel general continúa siendo minoritaria. La representación femenina apenas supera el $30 \%$ en los altos cargos de la Administración educativa y tampoco sobrepasa la predominancia masculina en la dirección de centros escolares. Se presenta una investigación Ilevada a cabo en dos institutos de secundaria de entorno rural y urbano de Castilla y León donde el acceso de las mujeres a la gestión se produce paulatinamente coincidiendo con la pérdida de prestigio y remuneración de los mismos. Con el fin de descubrir conocimientos y actitudes del alumnado ante el liderazgo femenino y sus particularidades se utiliza una metodología mixta, manejando como instrumentos de investigación la observación participante y una encuesta a 600 adolescentes. En los resultados detectamos que los estereotipos siguen presentes en el ideario estudiantil. Se descubre un estilo organizativo diferente por parte de las mujeres que pasa desapercibido para buena parte del alumnado. La conciencia de las dificultades en función del sexo es limitada, pero aumenta con la edad y en el sector femenino. Las chicas perciben de forma más clara los problemas añadidos de las mujeres para acceder a un cargo, mantenerlo y compaginarlo con la vida familiar.
\end{abstract}

PALABRAS CLAVE: Liderazgo femenino, organización escolar, género, gestión de centros educativos.

\section{ANALYSIS OF FEMALE LEADERSHIP FROM THE PERSPECTIVE OF THE STUDENTS IN TWO SECUNDARY SCHOOLS IN CASTILLA Y LEON}

ABSTRACT: In general terms, the participation of women in managing positions continues to be a minority. Female representation barely exceeds 
$30 \%$ in the high levels of the Educational Administration and does not surpass the male power in the control of schools. A research has been carried out in two secondary schools (rural and urban) of Castille and Leon where the access of women to the managing teams is taking place gradually coinciding with the loss of prestige and remuneration of the same ones. In order to check the knowledge and attitudes of the students about the female leadership and how it is different with regard to the male leadership, a mixed methodology has been used by developing a daily register and a survey to 600 teenagers. Studying the results we realize that stereotypes are still present in the young people's ideology, that different kind of leadership between men and women aren't rarely recognized by pupils. The awareness of difficulties is limited but increases among older students and the female sector. Girls perceive more clearly the added problems of women to access, maintain and reconcile a position with their family life.

KEYWORDS: Female leadership, school organization, gender, school management.

Recibido: 27/02/2017

Aceptado: 14/07/2017

Correspondencia: Sofía Díaz de Greñu Domingo, Universidad de Valladolid, Campus María Zambrano, Plaza de la Universidad, 40005 Segovia. Email: sofiadiazdegrenu@ gmail.com.

\section{MUJERES Y PARTICIPACIÓN EN ÓRGANOS DE GESTIÓN EDUCATIVOS}

En la cumbre mundial sobre Desarrollo Social de Copenhague en 1995 se adoptó el compromiso de asegurar la plena igualdad entre hombres y mujeres. El tercer objetivo hacía referencia al empoderamiento de las mujeres, término surgido de los movimientos feministas en América Latina para referirse al su grado de participación en los órganos democráticos de gobierno y estaba orientado a los Ilamados países pobres (Duflo, 2012). Como señalaba López Méndez (2005), aquellos países que no se englobaban en el citado ámbito parecían no tener obligaciones de acción o evaluación. En los denominados países ricos las mujeres, poco a poco, van incorporándose a las estructuras de poder, pero de forma limitada y con grandes obstáculos a la toma de decisiones (De Barbieri, 1992).

La inclusión de las mujeres en los puestos de gestión en los países de la Organización para la Cooperación y el Desarrollo Económicos (OCDE) es minoritaria. Un ejemplo lo tenemos en España donde en el año 2000 solo había un 2\% de mujeres en los puestos de alta dirección empresarial y en 2015 apenas se alcanza el 10\% quedando lejos de la recomendación del 40\% que recogía la Ley Orgánica para la Igualdad de Hombres y Mujeres (PriceWaterhouseCooper, 2015; Sarriés y Del Olmo, 2006). En la Administración Pública se presuponían datos más optimistas, dado que desde la Unión Europea se promovían acciones y porque existe una ma- 
yor presencia femenina en estos ámbitos (Comisión Europea, 2010). No obstante, tampoco alcanzan actualmente cotas paritarias en lo que se refiere a cargos de alta gestión, rondando el 30\% en los puestos de la Administración General del Estado, a pesar de que en 2011 se aprobó el I Plan de Igualdad entre Mujeres y Hombres en la Administración General del Estado y sus Organismos Públicos (Consejo Económico y Social, 2011; Instituto Nacional de Estadística, 2015).

En el campo de la docencia, las mujeres se situaban a finales del siglo XX en torno al 95\% en Educación Infantil (EI), 70\% en Educación Primaria (EP), 50\% en Educación Secundaria (ES) y Formación Profesional (FP), siendo algo superior el porcentaje en los centros privados. Es alrededor del año 2000 cuando se establece el equilibrio entre el número de mujeres y hombres docentes en la enseñanza secundaria. En años sucesivos las cifras apenas varían, según los datos del Ministerio de Educación y del Consejo Escolar del Estado (2015). Por otra parte, las mujeres están presentes como alumnas entre el 50 y el 60\% dependiendo de niveles y ramas de estudio. Estas cifras tienen que analizarse teniendo en cuenta que la enseñanza, especialmente en los primeros niveles, se asocia a la atención a los demás y la ética de cuidados asignada históricamente a las mujeres (Álvarez, 2011; Acker, 1995; Medina-Vicent, 2016) y que frenan la construcción de la igualdad real (Savigny, 2014).

En los órganos de decisión del ámbito educativo, tales como el Consejo Escolar del Estado y los Consejos Escolares Autonómicos, la representación femenina continúa siendo inferior a la de los hombres. Si realizamos un análisis de la evolución en la última quincena podemos señalar que en el año 2000 había un 13\% de mujeres frente a $87 \%$ de hombres, de un $17,64 \%$ en la alta inspección y de un $30 \%$ en los altos cargos de la Administración Educativa (Grañeras, 2004; Mañeru y Grañeras, 2005). En 2010 los porcentajes de mujeres en estos órganos se habían incrementado un 3\% de media (Ver Figura 1) (Centro de Investigación y Documentación Educativa, 2012; Consejo Escolar, 2015; Ministerio de Educación 2014a).

\section{Participación femenina en la alta administración educativa}

(\%)

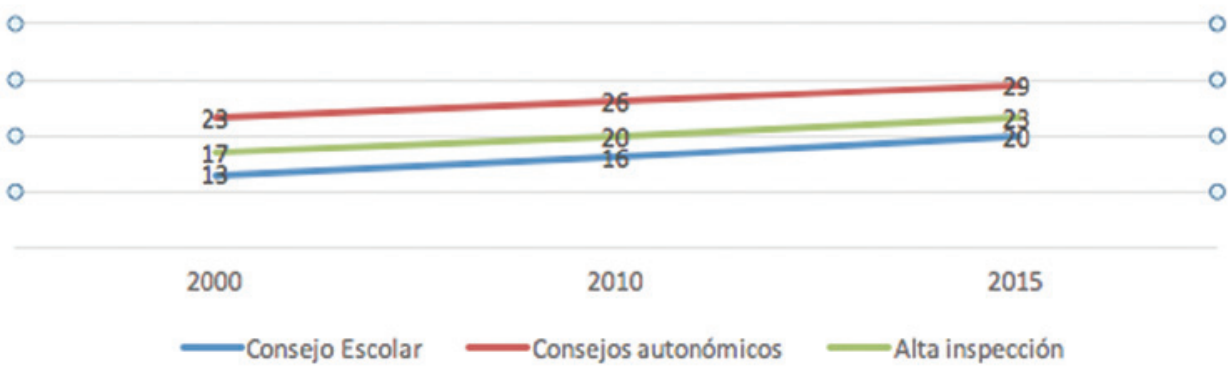

Figura 1. Evolución de la participación femenina en la Administración educativa. Fuente: Elaboración propia a partir de Centro de Investigación y Documentación Educativa, 2012; Consejo Escolar, 2015; Mañeru y Grañeras, 2005 
En cuanto a la ocupación de cargos directivos de centros escolares por parte de las mujeres la tendencia es al ligero aumento, pero no alcanza la misma proporción que el número de mujeres docentes. En el año 2000 en los niveles de Educación Infantil (EI) y Educación Primaria (EP) había un 45\% de directoras, un 59\% de jefas de estudio y un $52 \%$ de secretarias. Por tanto, el número de directoras era inferior al de directores, el de jefas de estudio y secretarias algo mayor, pero alejado del $70 \%$ de la presencia femenina en esos niveles.

En los centros de Educación Secundaria se registraba un 25\% de directoras, un $37,66 \%$ de jefas de estudio y un $36,19 \%$ de secretarias, estando las mujeres poco representadas en lo que se refiere a la dirección (Grañeras, 2004). En el conjunto del país solo una cuarta parte de los personas que desempeñaban la dirección de centros de secundaria era mujer (Grañeras, 2004, p. 636; Mañeru y Grañeras, 2005). Estas cifras pueden compararse a las de otros países vecinos como Francia donde la dirección de institutos recaía en un 26,4\% de mujeres (Rosenwald, 2005).

Quince años después en los equipos directivos en España se ha experimentado un incremento en las cifras. Para los centros de ES tenemos un 32\% de directoras, un $42 \%$ de secretarias y un $45 \%$ de jefas de estudio (CIDE, 2012; Ministerio de Educación, 2014a), pero también hay que tener en cuenta que el porcentaje de mujeres docentes en esos niveles se ha incrementado ligeramente, dado que el número de mujeres aumenta 14 puntos y el del acceso a los equipos 21 puntos (Ver Figura 2). Sin embargo, aún se halla una diferencia importante en lo que se refiere a la dirección, que es el cargo de máxima responsabilidad.

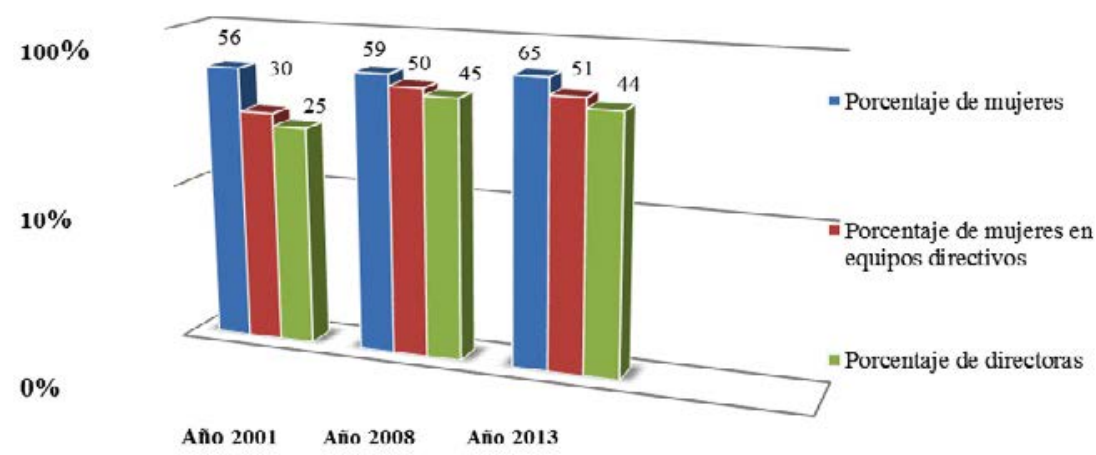

Figura 2. Representación de mujeres en los equipos directivos de centros de Educación Secundaria en España (2001-2013). Fuente: Elaboración propia a partir de datos en: Mañeru y Grañeras, 2005, pp. 13-84; Ministerio de Educación, 2008; Ministerio de Educación, 2014

Por tanto, diez años después siguen siendo válidas las reflexiones de Mañeru y Grañeras (2005) cuando señalaban que "El grado de representatividad de las mujeres en los órganos de dirección escolar, aunque ha aumentado en los últimos años, sigue sin guardar proporción con su presencia como docentes en todos los niveles educativos" (p. 15). 
Sería interesante realizar una comparativa por comunidades autónomas y analizar las diferencias que existen entre el entorno rural y el urbano, ya que en la dirección de los centros urbanos suele haber una mayor proporción masculina (Consejo Escolar, 2015). En Castilla y León se registran datos similares a los de España y los países de la OCDE. La cifra de mujeres en los equipos de secundaria está en torno al 59\%, aunque los puestos de dirección este porcentaje disminuye notablemente (Instituto de la Mujer, 2011).

Así mismo hay que tener en cuenta que estos cargos han perdido prestigio en los últimos quince años, pese a la responsabilidad e importancia que conllevan, y que en el $70 \%$ de los casos la Administración ha tenido que intervenir con nombramientos de oficio ante la ausencia de candidaturas (Azumendi, 2009; Carrasco, 2002; Coronel, Carrasco y Moreno, 2012). Estos cargos no han sido suficientemente reconocidos ni remunerados, por lo que el interés por ocuparlos ha ido decayendo (Zurutuza, 2009; Fundación Sociedad Europea y Educación, 2015). Por otra parte, nos encontramos con que la progresiva feminización de la enseñanza puede conllevar un desprestigio de la misma.

El hecho de que la enseñanza esté considerada una profesión feminizada tampoco supone ningún privilegio para las mujeres porque vemos que incluso hay quien se permite valorar como negativo para la educación...el que la mayoría de las personas dedicadas a la enseñanza sean mujeres. Socialmente no es una profesión bien considerada, de hecho, las profesiones pierden categoría a medida que son ocupadas por mujeres". (Coronel, Moreno y Padilla, 2002, p. 78)

Por último, tenemos que reseñar que hay estudios que han descrito las barreras que tienen las mujeres para acceder a los puestos de dirección (Díez, Terrón y Anguita, 2009) que tienen que ver con varios factores: por un lado, con la costumbre social, es decir, con el papel subalaterno y secundario asignado históricamente a las mujeres en los ámbitos sociales. Por otro lado, con la visión predominante de la dirección y el liderazgo ligado a un enfoque jerárquico e individualista, que poco tiene que ver con la experiencia vital y cultural de las propias mujeres. En este sentido, la barrera se fortalece, ya que para las mujeres la dirección escolar no es entendida como una conquista individual del poder sino como un servicio a la comunidad educativa y no se lo proponen como logro profesional, por lo tanto, sus expectativas bajan, dando por sentado que un hombre siempre se ajustará más al modelo de dirección que se espera colectivamente. Una última barrera tiene que ver con la falta de modelos femeninos en puestos de dirección con los que identificarse.

\section{ObJetivos, MEtOdología E INSTRUMENTOS DE INVESTIGACIÓN}

El alumnado que cursa estudios actualmente en la educación secundaria constituye el núcleo que formará parte de las estructuras sociales, laborales, económicas y políticas. Educar en igualdad significa no sólo recordar de vez en cuando los flagrantes casos de violencia de género que existen a nivel global, sino también enseñar a adoptar una postura crítica mostrando realidades que no causan alarma social, pero que se inscriben en un sistema que aún sigue siendo claramente discriminatorio para las mujeres. El ejemplo cotidiano de cómo se ejerce la dirección de los centros educativos es un elemento más de este proceso educativo y, por ello, necesitamos 
explorar la actitud de las nuevas generaciones, dado que nos ayudará a poner en marcha las acciones más adecuadas para transformar dichas realidades. Por otro lado, las instituciones escolares continúan educando a través del currículum oculto a los chicos para ejercer cargos de responsabilidad y a las chicas para desempeñar tareas de tipo secundario (Guramatunhu-Mudiwa y Bolt, 2012). Por ello y teniendo en cuenta el entorno en el que nos desenvolvemos profesionalmente, pusimos en marcha esta investigación que se planteaba como objetivos:

- Describir e interpretar la percepción del alumnado con respecto a la gestión femenina en relación a la masculina en centros de secundaria de la comunidad de Castilla y León.

- Reflexionar sobre los estilos de liderazgo relacionados con el género en los institutos de educación secundaria.

En Castilla y León encontramos un importante número de centros en el entorno rural, aunque el número de estudiantes que atienden es pequeño respecto a la población total, ya que los centros urbanos absorben buena parte de la demanda (Portal de Educación Castilla y León, 2016). Por ello, decidimos poner en marcha nuestro estudio en dos centros que abarcasen esta realidad: un centro de carácter rural y otro urbano.

A continuación, describimos brevemente los dos centros donde se llevó a cabo el estudio. El primer instituto, el centro rural, ofrece enseñanzas cofinanciadas por el Fondo Social Europeo, está organizado en tres líneas con una oferta educativa de Enseñanza Secundaria Obligatoria, Bachillerato de Ciencias y Tecnología y de Humanidades y Ciencias Sociales con una antigüedad de 27 años. En el curso 2016/17 atiende, aproximadamente, a 400 adolescentes procedentes no sólo de la localidad donde se sitúa, de 2300 habitantes, sino de otros núcleos cercanos con amplio porcentaje de población inmigrante ya asentada de forma definitiva. La actividad económica principal de la zona es la agricultura y ganadería. El equipo directivo está formado, desde hace tres cursos, por dos hombres y dos mujeres: el director, el jefe de estudios de bachillerato, la jefa de estudios de enseñanza secundaria obligatoria y la secretaria.

El segundo centro, urbano, está ubicado en una capital de provincia. Tiene una antigüedad de medio siglo aproximadamente, atiende a más de 1000 estudiantes y propone una oferta de Enseñanza Secundaria Obligatoria, Bachillerato (diurno, cinco líneas y nocturno, una línea), módulos a distancia de ambas etapas y un Ciclo Formativo de Formación Profesional. Atiende a alumnado del barrio donde está ubicado, pero también procedentes de la provincia y limítrofes, dado que es uno de los pocos centros de la comunidad con una oferta tan variada. El equipo directivo se compone, desde el curso pasado, del director, dos jefes de estudios (bachillerato y distancia) y tres jefas de estudios (secundaria, nocturno y formación profesional). El equipo anterior estaba integrado por un director, cuatro jefes de estudios y una jefa de estudios que continúa en el equipo actual. Como se observa, el puesto de máxima responsabilidad lo ostenta un hombre en ambos centros. Este hecho se repite con frecuencia en muchos institutos de esta provincia (Portal de Educación, 2016).

El enfoque metodológico que se ha seguido se basa en la metodología mixta; por un lado, se utiliza la observación participante bajo premisas etnográficas (Álvarez Álvarez, 2011; Salcedo, 2016) y, por otro, la técnica de la encuesta. 
La observación participante recogida en un diario de campo (Spradley, 1980) nos ha permitido a) detectar comportamientos adolescentes diferenciados ante la autoridad masculina y femenina; b) describir estilos de liderazgo de hombres y mujeres pertenecientes a los dos equipos directivos y c) detectar estereotipos y valorar la actitud de chicos y chicas ante la implementación de un cuestionario que describiremos seguidamente. Las observaciones se realizaron de forma sistemática durante los cursos 2014/15 (centro rural) y 2015/16 (centro urbano). Las sesiones de observación se produjeron con una periodicidad semanal de octubre a mayo en ambos cursos académicos (un total de 144 registros).

El cuestionario es una de las técnicas cuantitativas más empleada en investigación social (Cea D’Ancona, 2004; Hueso y Cascant, 2012). A pesar de desarrollarse para realizar sondeos previos de la situación, en nuestro caso cumplía la función de complementar las observaciones realizadas en el diario. Por ello, se construyó, validó y pasó una encuesta según propuestas de Grinnell y Unrau (2007). Se permitían respuestas cerradas afirmativas o negativas y se daba opción en todas las preguntas a respuestas libres de justificación (Anguita, Labrador y Campos, 2003). Este diseño ha permitido un análisis descriptivo cuantitativo y cualitativo (Castro y Godino, 2011) de las siguientes variables: 1) nivel (Educación Secundaria Obligatoria y Bachillerato), 2) sexo (mujer y hombre) y 3) entorno (rural y urbano) y alrededor de los siguientes elementos: a) el conocimiento del alumnado del acceso de mujeres y hombres a los puestos de dirección escolar; b) la importancia que se otorga al acceso de las mujeres a estos puestos; c) el establecimiento de jerarquías en relación al sexo; d) las diferencias de comportamiento percibidas ante la autoridad de un hombre y una mujer; e) a la opinión acerca de la influencia que pueda tener ocupar un cargo en la vida familiar y f) la valoración de estos cargos. Las preguntas se formularon teniendo en cuenta el nivel del alumnado y su desconocimiento sobre la normativa que rige el funcionamiento de los órganos de gobierno de los centros escolares y fue validado para asegurarnos de que las preguntas eran bien comprendidas por el grupo adolescente.

En el primer centro se pasó el cuestionario en un curso de cada nivel en la ESO y Bachillerato (200 estudiantes), mientras que, en el segundo, se implementó con una muestra significativa (400 estudiantes), alcanzando un nivel de confianza en torno al $95 \%$, con un margen de error del 5\%. Se establecieron cuotas para que cada centro, sexo y nivel estuviesen suficientemente representado. La selección de participantes fue aleatoria (Morales, 2012). El proceso seguido se resume en la Figura 3:
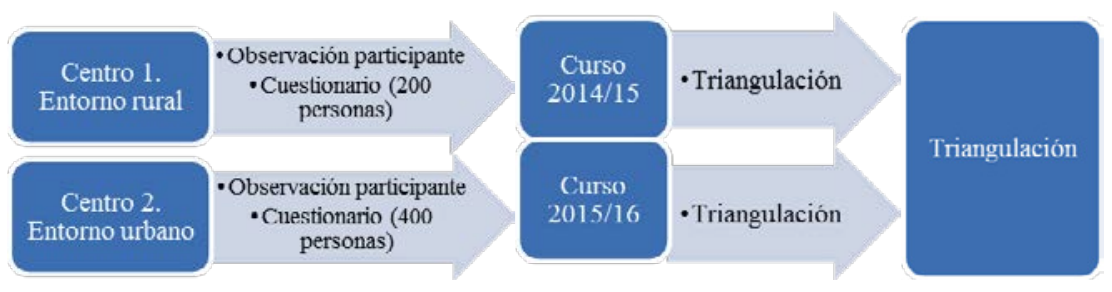

Figura 3. Contexto de trabajo y proceso de investigación. Cursos 2014/15 y 2015/16. Fuente: Elaboración propia 
Los criterios de rigor se basaron, siguiendo tanto premisas cuantitativas (Cea D'Ancona, 2004; Hueso y Cascant, 2012) como cualitativas (Guba y Lincoln, 2002), en la relevancia de las cuestiones planteadas, la adecuación de las preguntas de investigación a los instrumentos, la elección de muestras representativas, la sistematicidad en la aplicación de las herramientas etnográficas, la triangulación de métodos (Denzin y Lincoln, 2005), la consistencia y coherencia de los datos obtenidos con los de otras investigaciones y la revisión de posibles sesgos en la interpretación de resultados (Taylor y Bodgan, 2010).

En lo que se refiere a los criterios éticos, se adoptaron aquellos que están reconocidos por la comunidad científica internacional (American Psychological Association, 2010, pp. 11-13; Simons, 2011): protección de los derechos de los participantes, confidencialidad, obtención de permisos, reconocimiento de la propiedad intelectual, precisión y veracidad en la presentación de los datos, imparcialidad, equidad y compromiso.

\section{Resultados}

\section{Actitudes ante la feminización de los cargos y las diferencias de liderazgo en el medio rural y el medio urbano}

Durante el proceso de observación participante hemos constatado que los comportamientos adolescentes ante los profesores y las profesoras cambian significativamente. Debemos tener en cuenta que, en la actualidad, el principio de autoridad es rechazado, en líneas generales, especialmente a estas edades (Pereira, 2011). Sin embargo, se observan sistemáticamente mayores problemas de disciplina en las clases de las profesoras. Salvando las diferencias en cuanto a la personalidad y experiencia a la hora de mantener el orden en una clase, el alumnado desafía con más frecuencia la autoridad de las profesoras que de los profesores, detectándose varios episodios de enfrentamiento, incluso con las docentes de los equipos directivos. Por otro lado, los comportamientos disruptivos también están marcados por el género, al ser casi siempre protagonizados por alumnos.

La jefa de estudios ha tenido que intervenir en un aula porque uno de los chicos ha roto la persiana de la clase al manipularla de forma violenta. El adolescente niega ser el autor de los hechos y discute con la profesora y la representante del equipo directivo, a pesar de que el grupo ha sido testigo de su acción insiste en su actitud. "Yo solo quiero hablar con el director", dice". (Registro, número 56)

Por otra parte, los estilos de liderazgo de las mujeres de los equipos observados son diferentes a los de los hombres. Se aprecia, al margen de las peculiaridades de cada docente, una mayor empatía y carácter dialogante entre las mujeres (Sarriés y Del Olmo 2006): demuestran mayor disponibilidad y capacidad para resolver conflictos diplomáticamente e intentan conocer en profundidad los problemas emocionales del alumnado y de sus familias, les cuesta menos trabajar en equipo: "'es mejor hablar con su familia antes de proceder a amonestar', señala la jefa de estudios, 'quizá deberíamos tratar este asunto en la próxima reunión', 'es mejor sancionar rápidamente, sin tantas explicaciones', dice el jefe de estudios" (Registro número 20). 
Los profesores mantienen, en líneas generales, una actitud distante con el alumnado en el plano emocional, al menos en apariencia, y en ocasiones manifiestan actitudes paternalistas hacia sus compañeras y hacia las alumnas. Los profesores de los equipos observados delegan en sus compañeras tareas relacionadas con la mediación, la tutoría y las tareas burocráticas repetitivas: "te encargas de preparar los certificados, ¿vale? Eres ordenada y se te dan bien estas cosas" (Registro número 85). Suelen realizar bromas cuyo trasfondo machista está presente. "Haciendo similitudes entre el teclado del ordenador y un matrimonio: "la mujer es el Control y el hombre el Scape', comenta un profesor en tono jocoso" (Registro número 53). A veces las profesoras intentan hacérselo comprender, pero ellos mantienen que son comentarios inofensivos: "No se puede decir nada en estos tiempos, no tenéis sentido del humor" (Ibidem). Estos estilos detectados coinciden con los resultados de otras investigaciones realizadas en la misma línea (Carrasco, Coronel y Fernández, 2009).

El cuestionario y la introducción de los aspectos de igualdad en las clases fueron mejor acogidos por alumnas que por alumnos: "Un chico afirma: '¿por qué tenemos que hacer estas actividades? Se ensalza demasiado este tema'. Sus compañeras de la clase se han molestado con el comentario: 'parece mentira que todavía alguien siga pensando así con la de mujeres que están matando'" (Registro número 132). Las jóvenes hacen preguntas, atienden con gran interés, mientras que algunos de sus compañeros muestran desdén: "A mí esto me da igual" (Cuestionario número 425), la mayoría adopta una actitud discreta y no se pronuncia. Al final todo el mundo acepta realizar la encuesta y surgen debates espontáneos al respecto.

En cuanto a los datos cuantitativos, se realizan análisis a partir de las frecuencias de las respuestas afirmativas y negativas a los ítems del cuestionario descrito en el apartado anterior. Dichas frecuencias se manifiestan útiles para realizar previsiones y posteriormente reflexionar sobre los comentarios de carácter cualitativo que acompañan dichas respuestas (Anguita, Labrador y Campos, 2003; Hueso y Cascant, 2012). Del análisis de los datos (Tabla 1) (frecuencias marginales, es decir, el total del conjunto elegido) puede colegirse que en el medio rural la mayor parte del alumnado, sin haber diferencias significativas entre las variables sexo y edad, considera que el acceso a la dirección de los centros se produce prácticamente en igualdad de condiciones y argumenta que llegar a ocupar estos puestos depende de la capacidad y no del sexo. Por el contrario, según la opinión de la cuarta parte de la muestra, piensa que las dificultades continúan y señala que la paridad aún no se ha producido porque, utilizando una frase literal, "sigue habiendo machismo en la sociedad" (Cuestionario número 536) y los hombres tienen mayor facilidad para incorporarse a un equipo. El otro $75 \%$ explica que las diferencias en este caso concreto pertenecen al pasado.

Con respeto a la importancia que tiene llegar a ocupar estos cargos por parte de las mujeres, se constata una diferencia notable entre las respuestas de las chicas y las de los chicos. Si tenemos en cuenta las frecuencias marginales, un $87 \%$ de chicas frente a un $40 \%$ de chicos cree que es fundamental que las mujeres Ileguen a la dirección para conseguir la equidad (diferencia de proporción $r=p 1 / p 2=0,46$, la prueba de Pearson nos indica que la variable sexo en este caso sí tiene una dependencia con la respuesta afirmativa $\left.X^{2}=15,68\right)$. Este dato nos indica que las adolescentes intuyen cierto tipo de desigualdad que pasa inadvertida para los chicos: "Las mujeres tienen que demostrar que son capaces de hacerlo", señalan. (Cuestionario número 236) 
Tabla 1. Resultados del cuestionario sobre cargos directivos y género en el centro rural expresados en frecuencias relativas

\begin{tabular}{lccccccccc}
\hline & \multicolumn{4}{c}{ Sexo } & \multicolumn{5}{c}{ Nivel } \\
\cline { 2 - 10 } & \multicolumn{3}{c}{ Chico } & \multicolumn{2}{c}{ Chica } & \multicolumn{2}{c}{ ESO } & \multicolumn{3}{c}{ Bachillerato } \\
\cline { 2 - 9 } & Sí (\%) & No (\%) & Sí (\%) & No (\%) & Sí (\%) & No (\%) & Sí (\%) & No (\%) \\
\hline Dificultades acceso & 30 & 70 & 19 & 81 & 26 & 74 & 23 & 77 \\
Importancia issue & 42 & 58 & 87 & 13 & 77 & 23 & 70 & 30 \\
Liderazgo & 42 & 58 & 40 & 60 & 31 & 69 & 42 & 58 \\
Jerarquía correcta & 11 & 88 & 9 & 91 & 4 & 96 & 11 & 89 \\
Respeto autoridad & 64 & 36 & 73 & 27 & 84 & 16 & 68 & 32 \\
Conciliación & 32 & 68 & 58 & 42 & 46 & 54 & 43 & 57 \\
Valoración cargos & 32 & 68 & 35 & 65 & 36 & 64 & 33 & 67 \\
\hline
\end{tabular}

Fuente: Elaboración propia a partir de los datos obtenidos.

No hay diferencias significativas con respecto a las variables sexo y nivel en cuanto a la percepción en el cambio en las formas de liderar en el centro educativo. El $60 \%$ no aprecia diferencias entre el liderazgo masculino y femenino: "da igual que sea un hombre que una mujer" (Cuestionario número 528). Apenas alrededor de un $40 \%$ del alumnado detecta diferencias entre el estilo de gobierno de una mujer y el de un hombre. Aparecen ciertos comentarios entre las personas que perciben diferencias de estilo aunque, a veces, procedan de un pensamiento estereotipado: "las profesoras nos escuchan mejor si tenemos algún problema" (Cuestionario número 126); "las mujeres son más organizadas, escuchan con atención, son comprensivas" (Cuestionario número 325), "respetan los horarios", "cuidan el orden", "cumplen las normas", "nos obligan a limpiar la clase", etcétera (Cuestionarios números 5, 28, 173, 460). Estudios realizados en el contexto norteamericano también ponen de manifiesto que no hay apenas discrepancias, atendiendo a la variable sexo, en la percepción del liderazgo (Guramatunhu-Mudiwa y Bolt, 2012).

En cuanto al sistema de jerarquías con respecto al sexo, si bien en la etapa adolescente es difícil conocer las funciones que asigna el Reglamento Orgánico de Institutos de Enseñanza Secundaria (1996) a los órganos de gobierno unipersonales en un centro educativo, resulta Ilamativo que las chicas en mayor número coloque por orden correcto la jerarquía (dirección, jefatura de estudios, secretaría, jefaturas de estudios adjuntas), independientemente del sexo.

Por otra parte, alrededor de un $70 \%$ afirma que el respeto a la autoridad no se ve alterado por el sexo de los integrantes de los equipos. El 30\% restante apenas aduce razones para explicar la falta de consideración y acatamiento que se produce en ciertas ocasiones y no lo relaciona con el factor sexo, sino más bien con el carácter de las personas que han de ejercer dicha autoridad. Este dato se contradice parcialmente las observaciones realizadas.

En referencia a la compatibilidad de la vida familiar y personal también se aprecia una diferencia entre chicos y chicas. Aproximadamente un $65 \%$ de alumnas frente a un $35 \%$ de alumnos, $\left(r=0,53, X^{2}=8,38\right)$ expone que la vida familiar se ve alterada 
porque son las mujeres las que se encargan de las tareas del hogar. Los chicos no percibieron de forma tan evidente este hecho, aunque aquellos que optaron por el sí justificaban su respuesta señalando que la función de las mujeres como madres sí podría influir.

En lo referente al interés por ocupar este tipo de cargos, solo un 30\% del alumnado expresa su deseo de hacerlo, lo que indica la escasa valoración de dichos puestos en la actualidad. Algunos comentarios lo ponían de manifiesto: "porque es un trabajo odioso, los niños no respetan a nadie y no se gana mucho dinero" (Cuestionario número 328).

Para terminar, la valoración de la profesión docente en general y de los cargos de gestión de centros educativos en particular es bastante negativa, sobre todo en el caso de los chicos: "me gustaría dirigir una empresa pero no un colegio o un instituto" (Cuestionario número 450), "no me gusta que me falten al respeto, prefiero otras profesiones" (Cuestionario número 203). A medida que avanzamos en edad se adquiere mayor conciencia de desigualdad entre las adolescentes: "me gustaría ocupar un puesto de dirección pero siempre habrá alguien machista que me lo impida" (Cuestionario número 405), "el patriarcado nos oprime", "no quiero piropos, quiero respeto" (Cuestionario número 582), "las mujeres lo tienen mucho más difícil, casi siempre son ellas las que cuidan de los niños" (Cuestionario número 186). Estas valoraciones coinciden con otras investigaciones en el ámbito anglosajón (Coleman, 2007).

Tanto en las observaciones como en las respuestas a preguntas abiertas de la encuesta aparecen comentarios que muestran diferentes estereotipos relacionados con las atribuciones de género que tradicionalmente se han asignado al sexo femenino: "a las mujeres les afecta todo mucho más", "se alteran con facilidad", "las chicas seducen a los jefes con sus vestidos", "las mujeres están más capacitadas para ser profesoras que directoras", "las mujeres suelen ser más pacíficas", "hay mujeres que guardan rencor a los hombres y van a por ti a la mínima", "las mujeres no deberían ser directoras porque eso las quita de sus obligaciones como mujer".

Estos datos no difieren en gran medida entre el alumnado del centro rural y del centro urbano. Si observamos la Tabla 2, encontramos una ligera mayoría del medio urbano que piensa que la dificultad para acceder depende más de la capacidad y la formación que del sexo. Las personas que responden que sí existe relación con el sexo argumentan que aún sigue existiendo cierta discriminación que hace más difícil a las profesoras el acceso: "en mi colegio mandaba un chico", "sí porque solo hay directores hombres", "sigue habiendo machismo" (Cuestionarios números $228,304,414)$. Las chicas dan más importancia a la pregunta de investigación; se considera, en líneas generales, que se trata de un avance en la conquista de la igualdad: "porque las profesoras tienen los mismos derechos que los profesores" (Cuestionario número 312).

Por otra parte, aunque en el centro urbano hay un mayor número de respuestas con la jerarquía correcta, sigue apreciándose un porcentaje importante de personas (más del 50 \%) que ordena el rango en función del sexo de la persona, en este caso coloca al secretario por delante del jefe de estudios, mientras que en el centro rural a la secretaria se la colocaba la última por detrás de los jefes de estudios adjuntos. 
Tabla 2. Resultados del cuestionario sobre cargos directivos y género en el centro urbano expresados en frecuencias relativas

\begin{tabular}{lccccccccc}
\hline & \multicolumn{4}{c}{ Sexo } & \multicolumn{4}{c}{ Nivel } \\
\cline { 2 - 9 } & \multicolumn{2}{c}{ Chico } & \multicolumn{2}{c}{ Chica } & \multicolumn{2}{c}{ ESO } & \multicolumn{2}{c}{ Bachillerato } \\
\cline { 2 - 9 } & Sí (\%) & No (\%) & Sí (\%) & No (\%) & Sí (\%) & No (\%) & Sí (\%) & No (\%) \\
\hline Dificultades de acceso & 35 & 65 & 40 & 60 & 48 & 52 & 47 & 53 \\
Importancia issue & 40 & 60 & 80 & 20 & 63 & 47 & 82 & 18 \\
Liderazgo & 20 & 80 & 25 & 75 & 16 & 84 & 17 & 83 \\
Jerarquía correcta & 45 & 55 & 40 & 60 & 42 & 58 & 38 & 62 \\
Respeto a la autoridad & 70 & 30 & 74 & 26 & 62 & 38 & 72 & 28 \\
Conciliación & 49 & 50 & 55 & 45 & 45 & 55 & 50 & 50 \\
Valoración de cargos & 7 & 93 & 8 & 92 & 4 & 96 & 2 & 98 \\
\hline Funyyyyyyyyyyy
\end{tabular}

Fuente: Elaboración propia a partir de los datos obtenidos.

Las chicas tienen mayor conciencia de las dificultades para ejercer la autoridad por parte de las mujeres $\left(r=0,35\right.$, en este caso al ser $X^{2}=0,58<1$ no se puede establecer una relación de dependencia con la variable sexo aunque cuantitativamente sea mayor). Sin embargo, reflejan cualitativamente en sus respuestas la responsabilidad añadida de las mujeres en el ámbito privado, especialmente si tienen hijos: "eso se solucionaría si los hombres también cuidaran de la casa y los hijos", "en la limpieza solo hay mujeres" (Registro número 98). La valoración de este tipo de cargos es negativa salvo excepciones, tanto de los porcentajes como de los comentarios.

Los datos del centro urbano, por tanto, reflejan resultados parecidos a los del centro rural, aunque hay un ligero aumento en el número de chicas que sí son conscientes de la discriminación en el acceso, mayor número de personas de ambos sexos que no observan diferencias de liderazgo (al menos si atendemos a sus respuestas negativas y no a sus comportamientos o comentarios) y una mayor desvalorización de los cargos de gestión de centros docentes.

\section{Conclusiones}

Como ya se expresó en el apartado introductorio, pensamos que es necesario seguir reflexionando sobre la situación de la función directiva femenina en centros educativos en general y en los de Enseñanza Secundaria en particular, profundizando en el análisis de la percepción de la comunidad educativa, tal como se realiza en recientes trabajos al respecto (Coronel, Carrasco y Moreno, 2012).

A pesar de las limitaciones de tiempo y de contexto de este estudio, hemos podido descubrir que el acceso de las mujeres a los equipos directivos está lejos la equiparación en su presencia en la docencia no universitaria. Las investigaciones revisadas muestran que el estilo de liderazgo en instituciones escolares varía en función del sexo (Calvo y Rodríguez-Hoyos, 2012; Díez, Terrón y Anguita, 2006). Sin embargo, hallamos en este estudio una cierta disparidad: mientras que las observaciones y reflexiones en las respuestas abiertas lo corroboran (mayor empatía, implicación 
emocional y flexibilidad en el caso de las mujeres), un 80\% del alumnado declara en las respuestas de la encuesta no detectar estas diferencias. En este sentido, también existen estudios que apuntan hacia un estilo personal que no depende del sexo y es flexible (Eagly, Johannesen-Schmidt y Van Engen, 2003; Martin, 2011). En este caso, no es general en los estilos observados. Se percibe más bien en el sector femenino un estilo de dirección ya descrito por Carrasco, Coronel y Fernández (2009) con el que se suele crear cohesión social y buen ambiente de trabajo, dado que fomenta la participación, la flexibilidad y los modelos reticulares, descentralizados y democráticos y se atribuye a las mujeres mayores capacidades comunicativas y sociales.

El grupo de jóvenes participantes piensa, en su mayoría, que la igualdad ya se ha conseguido, aunque las chicas creen en mayor medida que es necesario avanzar en este terreno. Queda aún bastante camino para que la conciencia de ciertas manifestaciones de protesta ante la desigualdad, procedentes sobre todo de chicas en niveles de Bachillerato, se generalice al conjunto del alumnado y se reivindique abiertamente el feminismo, que no es más que un principio ético universal que defiende la igualdad entre hombres y mujeres. A pesar del creciente rechazo a la autoridad del profesorado en todo el colectivo, siguen apareciendo muestras de mayor consideración y acatamiento de instrucciones de profesores varones. Este hecho no se reconoce abiertamente, pero se percibe claramente durante las observaciones.

Los años pasan y Rodríguez Martínez (2011) nos recuerda que los códigos de género siguen arraigados en la institución escolar y marcan diferentes oportunidades laborales y sociales. Pese a una apariencia de igualdad (equiparación formal y legal), las dificultades persisten y las presiones en los cargos de gestión aumentan en el caso de las mujeres (Clavo y Goicoechea, 2013), tal y como hemos podido mostrar en este trabajo. Recientes estudios a nivel internacional muestran un liderazgo que se adapta a diferentes circunstancias y el contexto está marcado por los patrones de género (FuIler, 2014). El liderazgo se ha convertido en un importante discurso en el nuevo milenio y entenderlo desde una perspectiva de género es fundamental (Blackmore, 2013).

\section{REFERENCIAS BIBLIOGRÁFICAS}

Álvarez, C. (2011). El interés de la etnografía escolar en la investigación educativa. Estudios pedagógicos, 37, 267-279.

Acker, S. (1995). Carry on caring: the work of women teachers. British Journal of Sociology of Education, 16, pp. 21-36.

Álvarez, P. (2 de mayo de 2011). El rectorado es cosa de hombres. El País. Recuperado de: http://elpais.com/diario/2011/05/02/sociedad/1304287208_850215.html.

American Psychological Association (2010). Manual of the American Psychological Association. México: El Manual Moderno.

Anguita, J. C., Labrador, J. R. y Campos, J. D. (2003). La encuesta como técnica de investigación. Elaboración de cuestionarios y tratamiento estadístico de los datos (I). Atención primaria, 31(8), 527-538.

Azumendi, E. (22 de junio de 2009). ¿Por qué nadie quiere ser director de colegio? El País. Recuperado de: http://elpais.com/diario/2009/06/22/paisvasco/1245699599_850215. html. 
Blackmore, J. (2013). A feminist critical perspective on educational leadership. International Journal of Leadership in Education, 16(2), 139-154.

Calvo, A. y Rodríguez-Hoyos, C. (2012). Aportaciones de los estudios de las mujeres y del género a la organización escolar. Estado de la cuestión en España. Educación XXI, 15(1), 43-60.

Carrasco, M. J. (2002). El lado no visible del gobierno de los centros escolares. Ágora Digital, 4, 1-9.

Carrasco, M. J. (2004). Participación y poder de la mujer en las organizaciones educativas. Revista de Educación, 6, 75-87.

Carrasco, M. J., Coronel, J. M. y Fernández, M. L. (2003). Mujeres y dirección: la importancia de las relaciones como base de la política de gestión escolar. Investigación en la Escuela, 50, 5-20.

Castro, W. F. y Godino, J. D. (2011). Métodos mixtos de investigación en las contribuciones a los simposios de la SEIEM (1997-2010). En M. Marín, Fernández, Gabriel, Blanco, Lorenzo J. y Palarea, María Mercedes (Eds.), Investigación en Educación Matemática XV (pp. 99-116). Ciudad Real: Sociedad Española de Investigación en Educación Matemática, SEIEM.

Cea D'Ancona, M. Á. (2004). La senda tortuosa de la "calidad" de la encuesta. Reis, 111(5), 75-103.

Centro de Investigación y Documentación Educativa (2012). Mujeres en cargos de representación del Sistema Educativo II. Madrid Secretaría General Técnica. Servicio de publicaciones del Ministerio de Educación y Cultura.

Clavo, M. J. y Goicoechea, M. A. (2013). Miradas multidisciplinares para un mundo en igualdad. Contextos Educativos, 16, 173-174.

Coleman, M. (2007). Gender and educational leadership in England: A comparison of secondary headteachers' views over time. School Leadership and Management, 27(4), 383-399. http://doi.org/10.1080/1363243070.

Comisión Europea (2010). Carta de la Mujer. Recuperado de: http://www.upm.es/ sfs/ Rectorado/Gerencia/Igualdad/Documentos.

Consejo Económico y Social (2011). Tercer Informe sobre la situación de las mujeres en la realidad socio-laboral española. Madrid: CES.

Consejo Escolar del Estado (2015). Informe 2015 sobre el estado del sistema educativo. Recuperado de: http://www.mecd.gob.es/cee/publicaciones/informes-delsistema-educativo/informe-2015.html.

Corral, Y. (2009). Validez y confiabilidad de los instrumentos de investigación para la recolección de datos. Ensayo, 19(33), 228-247.

Coronel Llamas, J. M., Carrasco Macías, M. J. y Moreno Sánchez, E. (2012). Superando obstáculos y dificultades: un estudio multicaso sobre directoras escolares, políticas de liderazgo y gestión para la mejora. Revista de Educación, 357, 537-559.

Coronel Llamas, J. M., Moreno Sánchez, E. y Padilla Carmona, M. T. (2002). La gestión y el liderazgo como procesos organizativos: contribuciones y retos planteados desde una óptica de género. Revista de Educación, 327, 157-169.

De Barbieri, T. (1992). Sobre la categoría género. Una introducción teórico-metodológica. Revista interamericana de Sociología, 6(2-3), 147-178. 
Denzin, N. y Lincoln, Y. (Ed.) (2005). Handbook of Qualitative Research. Thousand Oaks, CA: Sage Publications.

Díez, J.; Terrón, E., Anguita, R. (Coord.) (2006). La cultura de género en las organizaciones escolares. Barcelona: Octaedro.

Díez, J., Terrón, E. y Anguita, R. (2009). Percepción de las mujeres sobre el techo de cristal en educación. Revista Interuniversitaria de Formación del Profesorado, $64(23,1), 27-40$.

Duflo, E. (2012). Women empowerment and economic development. Journal of Economic Literature, 50(4), 1051-1079.

Eagly, A. H., Johannesen-Schmidt, M. C. y Van Engen, M. L. (2003). Transformational, transactional, and laissez-faire leadership styles: A meta-analysis comparing women and men. Psychological Bulletin, 129(4), 569-591. http://doi.org/10.1037/00332909.129.4.569.

Fundación Europea Sociedad y Educación (2015). El prestigio de la sociedad docente en España. Percepción y realidad. Recuperado de: http://www.sociedadyeducacion.org

Grañeras Pastrana, M. (noviembre, 2004). Las mujeres en los equipos directivos en España. En J. López Yáñez, M. Sánchez Moreno y P. Murillo Estepa (Eds.), Cambiar con la sociedad, cambiar la sociedad. Actas del $8^{\circ}$ Congreso Interuniversitario de Organización e Instituciones Educativas (pp. 629-637). Sevilla: Secretariado de publicaciones de la Universidad.

Grinnell, R. M. y Unrau, Y. A. (2007). Social work research and evaluation: Foundations of evidence-based practice ( $8^{\mathrm{a}}$ Ed.). New York, NY: Oxford University Press.

Guba Egon, G. y Lincoln, Y. S. (2002). Paradigmas en competencia en la investigación cualitativa. En C. Denman y J. A. Haro (Comps.), Por los rincones. Antología de métodos cualitativos en la investigación social. El colegio de Sonora (pp. 113145). Sonora: Hermosillo.

Guramatunhu-Mudiwa, P. y Bolt, L. L. (2012). Does the gender of school personnel influence perceptions of leadership? School Leadership \& Management, 32(3), 261-277. http://doi.org/10.1080/13632434.2012.688742.

Hueso, A. y Cascant, M. J. (2012). Metodología y técnicas cuantitativas de investigación. Valencia: Universidad Politécnica.

Instituto de la Mujer (2011). Mujeres en cargos de representación del sistema educativo II. Madrid: Ministerio de Sanidad, Servicios Sociales e Igualdad.

Instituto Nacional de Estadística (2015). Mujeres en altos cargos públicos y privados. Recuperado de: http://www.ine.es/ss/Satellite.

López Méndez, I. (2005). Género en la agenda internacional de desarrollo. Un enfoque de Derechos Humanos. Revista Académica de Relaciones Internacionales, 2. Recuperado de: http://www.relacionesinternacionales.info.

Mañeru, A. (2004). Ser mujer y directora. En J. López Yáñez, M. Sánchez Moreno y P. Murillo Estepa (Eds.), Cambiar con la sociedad, cambiar la sociedad. Actas del $8^{\circ}$ Congreso Interuniversitario de Organización e Instituciones Educativas (pp. 665670). Sevilla: Secretariado de publicaciones de la Universidad. 
Mañeru, A. y Grañeras, M. (Coords.) (2005). Mujeres en cargos de representación del Sistema Educativo. Madrid: Instituto de la Mujer y Centro de Investigación y Documentación Educativa.

Martin, J. L. (2011). Toward a Conceptual Model of Feminist Leadership in American Education. In Jennifer L. Martin (Ed.), Women as leader in Education (pp. 1-37). Santa Bárbara: Michele A. Paludi, Series Editor.

Medina-Vicent, M. (2016). La ética del cuidado y Carol Gilligan: una crítica a la teoría del desarrollo moral de Kohlberg para la definición de un nivel moral postconvencional contextualista. Daimon. Revista Internacional de Filosofía, 67, 83-98. http://doi.org/10.6018/daimon/199701.

Ministerio de Educación, Cultura y Deporte (2008). Estudio para la distribución del profesorado de enseñanzas de régimen general no universitarias por enseñanza que imparte. Subsecretaría General Técnica. Oficina de Estadística. Recuperado de: http://www.mecd.gob.es.

Ministerio de Educación, Cultura y Deporte (2012). Datos y cifras del sistema universitario español. Subsecretaría General Técnica. Recuperado de: http://www.mecd. gob.es

Ministerio de Educación, Cultura y Deporte (2014). Estudio internacional de la enseñanza y el aprendizaje. Madrid: Secretaría General Técnica. Recuperado de: http://www.mecd.gob.es.

Ministerio de Educación, Cultura y Deporte (2014a). Datos y cifras. Curso escolar 2014/15. Subsecretaría General Técnica. Recuperado de: http://www.mecd.gob.es.

Morales, P. (2012). Tamaño necesario de la muestra: ¿Cuántos sujetos necesitamos? Estadística aplicada, 24. Recuperado de: http://web.upcomillas.es/personal/peter/ investigacion/Tama\%f1oMuestra.pdf.

Pereira, R. (2011). Adolescentes en el siglo XXI. Ediciones Morata.

Portal de Educación de Castilla y León (2016). Directorio de centros. Recuperado de: www.educa.jcyl.es.

PriceWaterhouseCoopers (2015). Las mujeres directivas en España. Recuperado de: https://www.pwc.es/es/publicaciones/gestion-empresarial/assets/la-mujer-directivaesp.pdf.

Reglamento Orgánico de Institutos de Enseñanza Secundaria (ROIE, 1996). Real Decreto 83/1996 de 26/01/96, pp. 17-40.

Wrushen, B. R. y Sherman, W. H. (2008). Women secondary school principals: Multicultural voices from the field. International journal of qualitative studies in education, 21(5), 457-469.

Rosenwald, F. (2005). Femmes aux postes de direction. Education \& Management, 29, 25-45.

Salcedo, W. N. (2016). Teoría crítica de la educación. Revista Paideia Surcolombiana, $11,75-84$.

Sarriés, L. y Del Olmo, N. (2006). Mujeres directivas en España. Estereotipos y realidad. Zaragoza: Mira editores.

Sarrió, M., Ramos, A., Barberá, E. y Candela, C. (2002). Mujeres directivas y demandas organizacionales. Revista de Psicología Social, 17(2), 183-192. 
Savigny, H. (2014). Women, know your limits: Cultural sexism in academia. Gender and Education, 26(7), 794-809.

Simons, H. (2011). El estudio de caso: Teoría y práctica. Ediciones Morata.

Spradley, J. P. (1980). Participant Observation. New York: Holt, Rinehart and Winston. Taylor, S. y Bogdan, R. (2010). Introducción a los métodos cualitativos de investigación. Buenos Aires. Paidós.

Zurutuza, I. (29 de septiembre de 2009). Nadie quiere dirigir el instituto. El País. Recuperado de: http://elpais.com/diario/2009/09/29/sociedad/1254175201_850215. html. 